\title{
Influence of scintillations on the performance of adaptive astronomical systems with Hartmann-like wavefront sensors
}

\author{
V. V. Voitsekhovich, V. G. Orlov, and L. J. Sanchez \\ Instituto de Astronomia, UNAM AP 70-264 Cd. Universitaria, 04510 Mexico D.F., Mexico
}

Received 10 November 2000 / Accepted 8 January 2001

\begin{abstract}
The influence of scintillations on image centroid measurements and on the phase reconstruction from Hartmann-like wavefront sensors is investigated quantitatively by means of computer simulations. It is shown that under the conditions of astronomical observations, the magnitude of the effect is between $10 \%$ (for excellent seeing) and $18 \%$ (for poor seeing). However, because the magnitude of the effect increases with the increasing of the turbulence strength, one can expect that under the strong-turbulence conditions the influence of scintillations on the image centroid can be quite strong. So, starting from agiven turbulence strength, it can be impossible to make a successful phase reconstruction from image centroid measurements. It is also shown that the scintillations affect in a different and complicated way the reconstruction quality of different aberrations. Nevertheless one can notice some general tendencies: The scintillations affect more strongly the reconstruction quality of the tip-tilt and high-order aberrations than the reconstruction quality of intermediate aberrations.
\end{abstract}

Key words. atmospheric effects - instrumentaion: adaptive optics - techniques: high angular resolution

\section{Introduction}

Hartmann-like wavefront sensors are often-used in astronomical adaptive systems for measurements of turbulenceinduced wavefront distortions (Voitsekhovich et al. 1988; Rigaut et al. 1991; Jiang et al. 1993; Li et al. 1993; Colucci et al. 1994; Rigaut et al. 1997). These sensors are popular in applications because they provide a direct and simple relationship between the measurements and phase gradients at the telescope aperture: It is assumed that the phase gradient averaged over a subaperture of a Hartmann mask is proportional to the corresponding image centroid offset. This simple relationship, however, is valid if the effect of amplitude fluctuations (scintillations) is not taken into account. It is widely accepted that under the weakturbulence conditions which are of main interest for astronomical observations, the effect of scintillations on the image centroid is negligible (Roddier 1981) but this assumption has never been supported by quantitative calculations.

In this paper we calculate the magnitude of the effect of interest by means of computer simulations. The simulations are based on the recently proposed method of random wave vectors (RWV) (Kouznetsov et al. 1997; Voitsekhovich et al. 1999) that allows us to simulate the

Send offprint requests to: V. V. Voitsekhovich, e-mail: voisteko@astroscu.unam.mx amplitude and phase samples with the desired statistics and cross-statistics.

\section{Effect of scintillations on a single image centroid}

The set of Hartmann measurements is composed from the measurements of separated image centroids. So, at first we consider the effect of scintillations on a single image centroid, and then we investigate how the scintillations affect the quality of phase reconstruction from Hartmann data.

Let the wave $\Psi(\boldsymbol{\rho})$ pass through a thin lens of diameter $d$ and focal length $f$. The centroid $\boldsymbol{\rho}_{\mathrm{c}}$ of the image formed by this wave at the lens focal plane can be written as (Tatarski 1968):

$\boldsymbol{\rho}_{\mathrm{c}}=\left\{x_{\mathrm{c}}, y_{\mathrm{c}}\right\}=-\frac{f}{k} \frac{\int_{G_{\mathrm{d}}} \mathrm{d}^{2} \rho \exp \{2 \chi(\boldsymbol{\rho})\} \boldsymbol{\nabla} S(\boldsymbol{\rho})}{\int_{G_{\mathrm{d}}} \mathrm{d}^{2} \rho \exp \{2 \chi(\boldsymbol{\rho})\}}$,

where $x_{\mathrm{c}}$ and $y_{\mathrm{c}}$ denote the Cartesian coordinates of image centroid, $\chi(\boldsymbol{\rho})$ and $S(\boldsymbol{\rho})$ are the log-amplitude and the phase of the wave $\Psi$, respectively, $k$ is the wavenumber, and $G_{\mathrm{d}}$ denotes the integration over the lens aperture.

However, in experiments related to the phase reconstruction from centroid measurements it is always assumed that the effect of amplitude fluctuations on the 


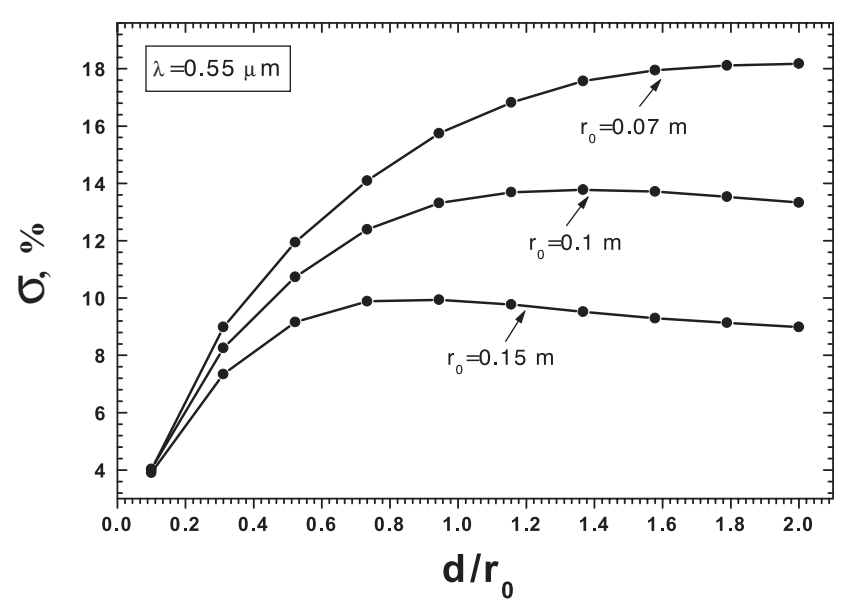

Fig. 1. Relative error $\sigma$ versus the ratio of lens diameter $d$ to the Fried parameter $r_{0}$

image centroid is negligible. Mathematically this assumption can be written as

$\boldsymbol{\rho}_{\mathrm{c}}^{\prime}=\left\{x_{\mathrm{c}}^{\prime}, y_{\mathrm{c}}^{\prime}\right\}=-\frac{f}{k \Sigma} \int_{G_{\mathrm{d}}} \mathrm{d}^{2} \rho \boldsymbol{\nabla} S(\boldsymbol{\rho})$,

where $\Sigma$ denotes the lens area.

Since in the problems related to propagation through atmospheric turbulence, the quantities $\boldsymbol{\rho}_{\mathrm{c}}$ and $\boldsymbol{\rho}_{\mathrm{c}}^{\prime}$ are random, we can define the relative error $\sigma$ of image centroid measurements associated with scintillations as

$\sigma=\frac{1}{2}\left[\frac{\sqrt{\left\langle\left(x_{\mathrm{c}}-x_{\mathrm{c}}^{\prime}\right)^{2}\right\rangle}}{\sqrt{\left\langle x_{\mathrm{c}}^{2}\right\rangle}}+\frac{\sqrt{\left\langle\left(y_{\mathrm{c}}-y_{\mathrm{c}}^{\prime}\right)^{2}\right\rangle}}{\sqrt{\left\langle y_{\mathrm{c}}^{2}\right\rangle}}\right]$.

From a physical point of view, the error $\sigma$ shows how big the relative contribution of scintillations is to the image centroid offset. In what follows, we calculate this error as a function of turbulence conditions and lens size.

In order to calculate the error $\sigma$ we apply the method of random wave vectors (RWV) (Kouznetsov et al. 1997; Voitsekhovich et al. 1999) that allows us to simulate the phase and log-amplitude samples with desired statistics and cross-statistics. The detailed description of RWV method and step-by-step simulation procedure can be found in Kouznetsov et al. (1997). The present simulations are performed for the Hufnagel model of $C_{n}^{2}$ profile (turbulence strength profile) that is given by (Hufnagel 1974)

$$
\begin{aligned}
C_{n}^{2}(z)= & C_{0} r_{0}^{-5 / 3} k^{-2} \\
& \times\left[\left(\frac{z}{z_{0}}\right)^{10} \exp \left\{-\frac{z}{z_{1}}\right\}+\exp \left\{-\frac{z}{z_{2}}\right\}\right],
\end{aligned}
$$

where $r_{0}$ is the Fried parameter, $k$ is the wavenumber, $C_{0}=1.02710^{-3} \mathrm{~m}^{-1}, z_{0}=4.63210^{3} \mathrm{~m}, z_{1}=10^{3} \mathrm{~m}$, $z_{2}=1.510^{3} \mathrm{~m}$.
Table 1. Relationship between Fried parameter $r_{0}$ and image $F W H M$

\begin{tabular}{|c|c|c|}
\hline \multicolumn{3}{|c|}{$\lambda=0.55 \mu \mathrm{m}$} \\
\hline $\begin{array}{c}\text { Fried } \\
\text { parameter, } \mathrm{m}\end{array}$ & $\begin{array}{c}\text { FWHM, } \\
\text { arcsec }\end{array}$ & $\begin{array}{c}\text { FWHM, } \\
\text { arcsec }\end{array}$ \\
\hline 0.07 & 1.60 & 1.58 \\
\hline 0.08 & 1.41 & 1.38 \\
\hline 0.09 & 1.25 & 1.23 \\
\hline 0.1 & 1.13 & 1.11 \\
\hline 0.11 & 1.03 & 1.01 \\
\hline 0.12 & 0.94 & 0.92 \\
\hline 0.13 & 0.87 & 0.85 \\
\hline 0.14 & 0.81 & 0.79 \\
\hline 0.15 & 0.76 & 0.74 \\
\hline
\end{tabular}

So, using Eqs. (1-3) and the samples simulated by the RWV method, we calculate the relative error $\sigma$ defined by Eq. (3). The number of samples used in the simulation is 5000. The simulation results are shown in Fig. 1 where the relative error $\sigma$ is plotted versus the ratio of lens diameter $d$ to the Fried parameter $r_{0}$. In Fig. 1 we show the error of interest for three cases of seeing conditions: poor seeing $\left(r_{0}=0.07 \mathrm{~m}\right)$, good seeing $\left(r_{0}=0.1 \mathrm{~m}\right)$, and excellent seeing $\left(r_{0}=0.15 \mathrm{~m}\right)$. Because in the astronomical community it is widely accepted to characterize the seeing by the image $F W H M$ rather than by the Fried parameter, we present a relationship between two parameters in Table 1. Table 1 includes two very different telescope diameters $(1 \mathrm{~m}$ and $10 \mathrm{~m})$ in order to stress that, for the considered range of telescope sizes, the relationship of interest is affected slightly by the telescope diameter.

As one can see from Fig. 1, the error initially grows to a maximum magnitude, and then slowly approaches to an asymptotic level. This behavior is mainly determined by the cross-correlation between the log-amplitude and phase gradient fluctuations that have the same salient features: it starts from zero, reaches its maximum magnitude under some separation between the observation points, and then starts to decrease. So, in the initial phase, while the lens size is smaller than the log-amplitude phase gradient cross-correlation length, the error increases because the log-amplitude and phase gradient fluctuations become more and more correlated with the increasing lens size. After reaching its maximum magnitude, the error starts to decrease due to the progressive decorrelation between the log-amplitude and phase gradient fluctuations. Physically, it means that the main contribution to the error comes from the aperture zones within which the log-amplitude and phase gradient are still correlated. With increasing lens size this contribution starts to be smaller and smaller, as is reflected in the behavior of the error. 


\section{Scintillation-induced error of phase reconstruction from Hartmann data}

In the Hartmann test a set of image centroid measurements $\mathbf{H}$ is used to reconstruct the phase at the aperture. In real experiments these measurements are always affected by scintillations that can be written mathematically as

$\mathbf{H}\left(\boldsymbol{\rho}_{s}\right)=-\frac{f}{k} \frac{\int_{G_{s}} \mathrm{~d}^{2} \rho \exp \{2 \chi(\boldsymbol{\rho})\} \nabla S(\boldsymbol{\rho})}{\int_{G_{s}} \mathrm{~d}^{2} \rho \exp \{2 \chi(\boldsymbol{\rho})\}}, s=1, \ldots, N,(5)$ where $\boldsymbol{\rho}_{s}$ denotes the center of $s$ th subpupil, $G_{s}$ denotes the integration over sth subpupil, $\chi$ and $S$ are the logamplitude and the phase, respectively, and $N$ is the number of subpupils.

However, in order to provide a successful phase reconstruction, one always uses a scintillation-free approximation $\mathbf{H}^{\prime}$ of Hartmann measurements that can be written as

$\mathbf{H}^{\prime}\left(\boldsymbol{\rho}_{s}\right)=-\frac{f}{k \Sigma_{s}} \int_{G_{s}} \mathrm{~d}^{2} \rho \boldsymbol{\nabla} S(\boldsymbol{\rho}), s=1, \ldots, N$,

where $\Sigma_{s}$ denotes the area of $s$ th subpupil.

So, there is always some scintillation-related error of phase reconstruction that arises from the difference between Eqs. (5) and (6). In our case, when the logamplitude and phase fluctuations are random, this error is a statistical quantity, and can be considered as a variance of the difference between the phases restored from the sets of scintillation-affected and scintillation-free Hartmann measurements. For the completeness of analysis it is preferable to calculate not only the total error of phase reconstruction, but also the error for each aberration that allows one to see the reconstruction quality of separated aberrations. Such an investigation can be done if, for example, the Zernike phase expansion is included in the analysis (Voitsekhovich 1996).

Expanding the phase $S(\boldsymbol{\rho})$ over the set of Zernike polynomials, one can write

$$
\begin{aligned}
S(\boldsymbol{\rho}) & =\sum_{i=2}^{\infty} a_{l} Z_{l}(\boldsymbol{\rho} / R), \\
a_{l} & =\frac{1}{\pi R^{2}} \int_{G_{R}} \mathrm{~d}^{2} \rho Z_{l}(\boldsymbol{\rho} / R) S(\boldsymbol{\rho}),
\end{aligned}
$$

where $Z_{l}$ are the two-dimensional Zernike polynomials (Noll 1976), $R$ is the telescope radius, and $G_{R}$ denotes the integration over the aperture.

The Zernike coefficients $a_{l}$ to be obtained from the Hartmann data $\mathbf{H}$ can be written as

$a_{l}=T[\mathbf{H}]$,

where the operator $T$ is a symbolic notation for the reconstruction procedure.

In the present paper we use the procedure of leastsquare fit to the phase gradient. In terms of the Zernike

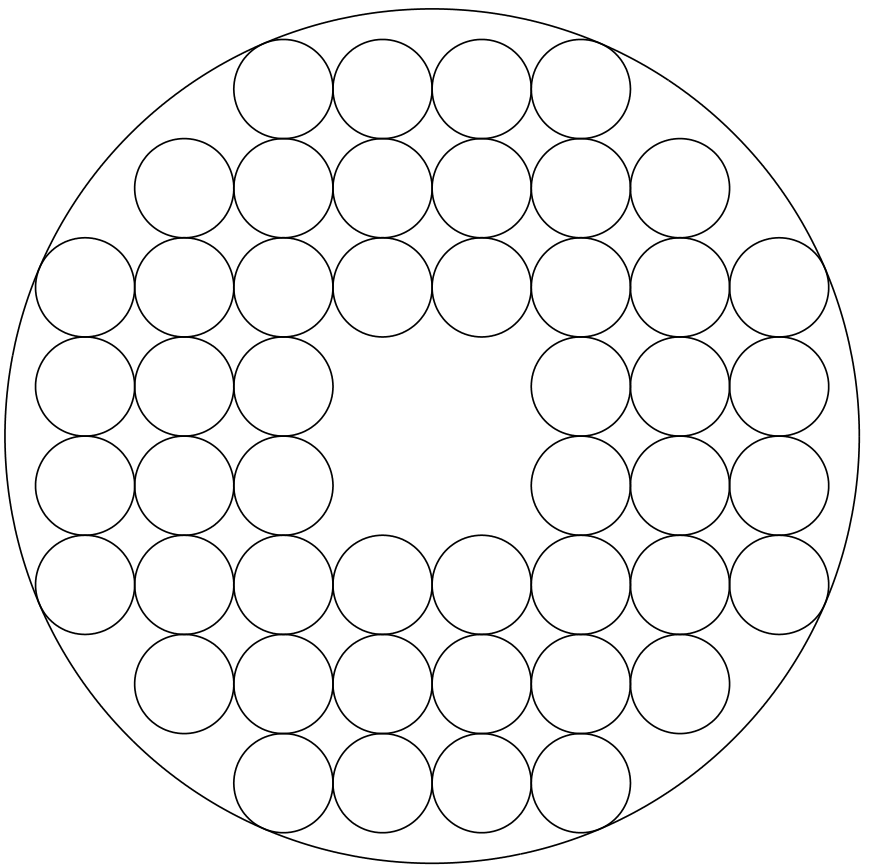

Fig. 2. Hartmann mask configuration

expansion, this procedure can be written as

$\sum_{s=1}^{N} \sum_{l=2}^{L}\left[a_{l} \boldsymbol{\nabla} Z_{l}\left(\boldsymbol{\rho}_{s} / R\right)-\mathbf{H}\left(\boldsymbol{\rho}_{s}\right)\right]^{2}=\min$.

So, denoting by $a_{l}$ and $a_{l}^{\prime}$ the Zernike coefficients restored from scintillation-affected $\left[\mathbf{H}\left(\boldsymbol{\rho}_{\mathbf{s}}\right)\right.$, Eq. (5)] and scintillation-free $\left[\mathbf{H}^{\prime}\left(\boldsymbol{\rho}_{\mathbf{s}}\right)\right.$, Eq. (6)] Hartmann data, respectively, one can write the relative scintillation-induced error $\sigma_{l}$ of $l$ th Zernike mode reconstruction as

$\sigma_{l}=\sqrt{\left\langle\left(a_{l}-a_{l}^{\prime}\right)^{2}\right\rangle /\left\langle a_{l}^{2}\right\rangle}$

The simulation procedure is as follows. First, we generate with the RWV method the log-amplitude and phase samples and calculate from these samples the scintillationaffected $\mathbf{H}\left(\boldsymbol{\rho}_{s}\right)$ and scintillation-free $\mathbf{H}^{\prime}\left(\boldsymbol{\rho}_{s}\right)$ Hartmann data using Eqs. (5) and (6), respectively. Then, applying the least-square procedure, we restore the Zernike coefficients $a_{l}$ and $a_{l}^{\prime}$ for each sampling. And finally, using Eq. (10) and averaging over the samples, we calculate the errors $\sigma_{l}$.

The simulation has been performed with a 48-subpupil Hartmann mask for a $2 \mathrm{~m}$ telescope diameter. The mask configuration is shown in Fig. 2, and the number of samples used in the simulation is 5000 . The simulation results are plotted in Fig. 3. As for the case of a single centroid error, we present the results for three cases of seeing conditions: poor seeing $\left(r_{0}=0.07 \mathrm{~m}\right)$, good seeing $\left(r_{0}=0.1 \mathrm{~m}\right)$, and excellent seeing $\left(r_{0}=0.15 \mathrm{~m}\right)$. One can see that the error depends in a complicated way on the Zernike mode number that is due to a different complexity of the geometric structure of Zernike polynomials. Nevertheless, one can notice some general tendencies. 


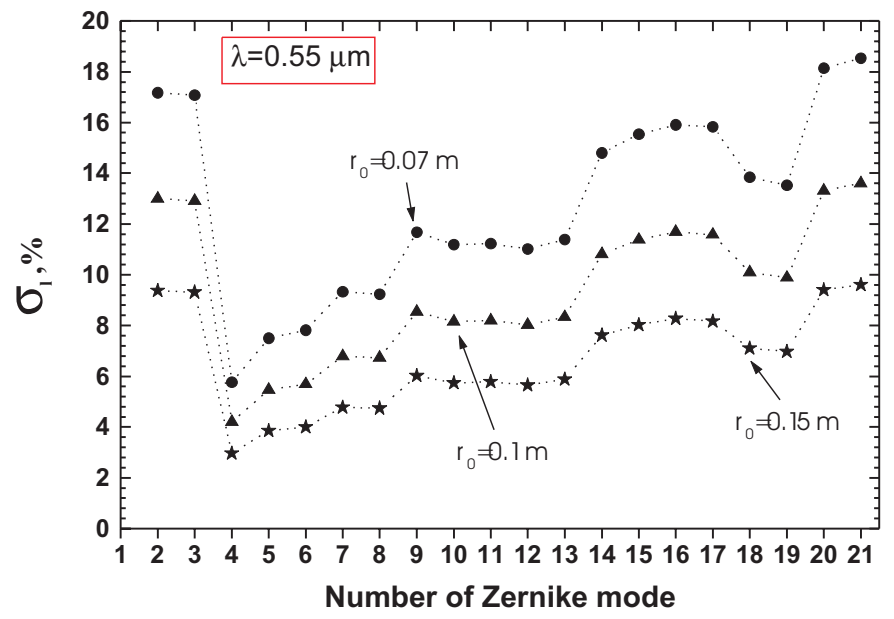

Fig. 3. Relative error $\sigma_{l}$ of Zernike mode reconstruction versus the mode number

For the lowest-order aberrations (tip-tilt, N2 and N3), the error is between 10-18\% (depending on the seeing conditions). Then, for the second-order aberrations (defocus and astigmatism, NN 4-6), it drops abruptly in 2-3 steps. With the increase in the aberration number, the error increases (with some oscillations), and for high-order aberrations it reaches practically the same magnitude as for the case of tip-tilt. So, generally speaking, the scintillations more strongly affect the reconstruction quality of the tip-tilt and high-order aberrations, while for intermediate aberrations the effect is less pronounced.

\section{Conclusions}

We have estimated quantitatively how scintillations affect the measurement of the image centroid. The results obtained show that, under the conditions of astronomical observations, the magnitude of the effect is between $10 \%$ (for excellent seeing) and 18\% (for poor seeing). The influence of scintillations on the quality of phase reconstruction from Hartmann data was also analyzed. The results shown that the scintillations affect in a different and complicated way the reconstruction quality of different aberrations. However, one can see a general trend: the scintillations more strongly affect the reconstruction quality of the tip-tilt and high-order aberrations than the reconstruction quality of intermediate aberrations.
In this paper we have applied the RWV method that was developed for investigations related to weakturbulence propagation. Because weak-turbulence conditions are of strong interest in astronomical observations, our quantitative analysis was restricted to this case only. Nevertheless, the results obtained allow us to draw some qualitative conclusions related to the strong-turbulence propagation that is of importance for a number of another applications. It was shown that the magnitude of the effect increases with increasing turbulence strength. So, one can expect that under the strong-turbulence conditions, the influence of scintillations on the image centroid can be quite strong, and starting from a given turbulence strength, it can make impossible a successful phase reconstruction from image centroid measurements. The quantitative results corresponding to the strong turbulence case can be obtained applying another simulation technique, for instance that based on propagation through multiple phase screens (Martin \& Flatte 1988).

\section{References}

Coluccib, D., Lloyd-Hart, M., Wittman, D., et al. 1994, PASP, 106, 1104

Hufnagel, R. E. 1974, in Optical Propagation through Turbulence, OSA Technical Digest Series, OSA, Washington, D. C., WA1-1

Jiang, W., Li, H., Liu, C., et al. 1993, in Proc. ICO-16 Satellite Conf. on Active and Adaptive Optics, ed. F. Merkle, ICO 16 Secretariat (Garching, Germany), 127

Kouznetsov, D., Voitsekhovich, V. V., \& Ortega-Martinez, R. 1997, Appl. Opt., 36, 464

Li, H., Xian, H., \& Jiang, W. 1993, in Proc. ICO-16 Satellite Conf. on Active and Adaptive Optics, ed. F. Merkle, ICO 16 Secretariat (Garching, Germany), 21

Martin, J. M., \& Flatte, S. M. 1988, Appl. Opt., 27, 2111

Noll, R. J. 1976, J. Opt. Soc. Am., 66, 207.

Rigaut, F., Rousset, G., Kern, P., et al. 1991, A\&A, 250, 280

Rigaut, F., Ellerbroek, B. L., \& Northcott, M. J. 1997, App. Opt., 36, 2856

Roddier, F. 1981, Prog. Opt., 19, 281

Tatarski, V. I. 1968, The Effects of the Turbulent Atmosphere on Wave Propagation, National Science Foundation Report TT-68-50464

Voitsekhovich, V. V. Gubin, V. B., \& Mikulich, A. V. 1988, Atmo. Opt., 1, 66

Voitsekhovich, V. V., Kouznetsov, D., Orlov, V. G., \& Cuevas, S. 1999, Appl. Opt. 38, 3985

Voitsekhovich, V. V. 1996, J. Opt. Soc. Am. A, 8, 1749 